\title{
IN MEMORIAM: PAUL ERDŐS
}

1913-1996

With the death of Paul Erdös on September 20, 1996, not only did the twentieth century lose one of its finest mathematicians, but the world lost one of the strongest ever to have appeared.

Erdős was born on March 26, 1913 to two mathematics teachers in Hungary, and almost immediately began a career in mathematics. At the age of four he discovered the negative numbers, and amused himself by multiplying four-digit numbers in his head.

By the age of 21 Erdős had received his Ph.D. from Pázmány University, where he was already exposed to logic, or at least to logicians. For example, in 1930 he found a simple proof of Chebyshev's theorem that for any integer $n$ there is always a prime between $n$ and $2 n$, and László Kalmár helped him to prepare his paper for publication.

Also, in 1932 George Szekeres, one of Erdős's friends, rediscovered Ramsey's Theorem and applied it to a problem posed by Eszter Klein. Ramsey's Theorem greatly impressed Erdös, and he spent much of his life pursuing applications of it and similar propositions. Already by 1935 he and Szekeres had a joint paper with applications of Ramsey's Theorem to graph theory.

From 1934 to 1938 Erdős was in Manchester, England and at approximately the same time Richard Rado received a scholarship to Cambridge University. Rado had finished his Ph.D. with Issai Schur in 1931 in Germany on a problem related to Ramsey theory. By 1933 it was already difficult for a Jew to find employment in Germany. Erdös and Rado met in Cambridge in 1934 and began a collaboration that changed the face of set theory. What they invented together was the partition calculus, a generalization of Ramsey theory to uncountable cardinals and infinite order types. The consequences of this theory are still being investigated.

In 1938 Erdös came to the United States, and gradually began the habit of traveling between institutions that eventually gained him his reputation as a sort of mathematical circuit rider.

Erdös's breadth of knowledge and achievement in mathematics was enormous. He studied large parts of number theory and combinatorics as well as set theory. It is impossible to find any bounds to his interests. Through his collaborations with hundreds of other people, he created new areas of study and new mathematical methods. For example, in number theory and combinatorics he developed the use of random methods (also used in parts 
of logic), and in set theory his name is inextricably linked with the partition calculus, and these are only two of his many great achievements. He published about 1500 papers with about 500 coauthors, and even now the exact number is unclear, for more joint papers are being published all the time.

He constantly sought out young people in mathematics, his "epsilons", by which he meant both child prodigies, especially in Hungary, and others just beginning a career. For example, in set theory he discovered Attila Máté and encouraged his work in set theory, which had as one consequence the writing of the book Combinatorial Set Theory: Partition Relations for Ordinals, by Erdős, Hajnal, Máté, and Rado.

Erdös began his work in set theory simply by extending some of the ideas and questions in Ramsey theory and graph theory to infinite sets and cardinals, a step he regarded as perfectly natural. His first result was joint with Dushnik and Miller. They showed that every graph of regular cardinality $\kappa$ with no infinite complete subgraph contains an independent set of size $\kappa$, and Erdös showed that this holds when $\kappa$ is singular as well. His proof appears as part of the Dushnik-Miller paper [1].

Soon after, in a paper [2] published in 1942, Erdős proved what is often referred to as the Erdös-Rado theorem, $\left(2^{\kappa}\right)^{+} \rightarrow\left(\left(\kappa^{+}\right)_{\kappa}^{2}\right.$ for all $\kappa$, although the arrow notation was not yet invented.

Erdős also worked with Tarski on large cardinals (mostly inaccessibles) and did some work on set mappings before publishing the great partition calculus paper with Rado in 1956 in which the general Erdös-Rado theorem (for partitions of finite subsets) was proved. This paper also introduced the familiar arrow-notation for partition relations which has become known in some quarters as "Erdős notation", but was actually invented by Rado, as was the term "partition calculus" itself. Erdős and Rado also succeeded in finding counterexamples to prove that their methods were optimal.

In 1955 Erdős met a young thesis student of Kalmár in Szeged, and the two got on so well together that they jointly published more than 50 papers, more than Erdös published with anyone else. That young student's name was Andras Hajnal. As he tells the story, when he and Erdös were first left alone, he spoke of his thesis work on relative constructibility, and was rather taken aback when Erdös responded to ask whether he was also interested in "normal" set theory. The two collaborated on problems both in the partition calculus and on related topics. Eventually, in 1965, the famous "Giant Triple Paper" [3] appeared, written by Erdős, Hajnal and Rado.

The triple paper answered many open questions about the partition calculus, but there was more to come. Much was connected with the subject of "Erdo"s cardinals", i.e., cardinals $\kappa$ with the property that $\kappa \rightarrow(\alpha)_{2}^{<\omega}$ for various values of $\alpha$. A visit by Hajnal to Berkeley in 1964 brought these 
to the attention of the set theorists and logicians there. One striking consequence was the work by Silver, Solovay, and others making use of these cardinals to derive the existence of $0^{\#}$ and in general to introduce the notion of indiscernibility into set theory and model theory.

Subsequently there has developed a great deal of work on large cardinals, and it seems to be accepted without question that many of these notions have equivalent forms in terms of the partition calculus.

But even the most elementary part of the partition calculus is not yet understood for countable ordinals. As he often did with problems that interested him, Erdös offered a large prize, actually one of his largest at $£ 1000$, for the complete characterization of all countable ordinals $\alpha$ satisfying $\omega^{\omega^{\alpha}} \rightarrow\left(\omega^{\omega^{\alpha}}, n\right)^{2}$ for all finite $n$. This prize is as yet unclaimed.

It appears that the work begun by Erdös in set theory will not be finished for some time.

JAMES E. BAUMGARTNER

\section{REFERENCES}

[1] B. Dushnik and E. W. MiLler, Partially ordered sets, American Journal of Mathematics, vol. 63 (1941), p. 605.

[2] P. ERDös, Some set-theoretical properties of graphs, Revista de la Univ. Nac. de Tucuman, Ser. A. Mat. y Fiz. Teor., vol. 3 (1941), pp. 363-367.

[3] P. Erdős, A. Hajnal, and R. Rado, Partition relations for cardinal numbers, Acta Mathematica Hungarica, vol. 16 (1965), pp. 93-196. 